\title{
A distribuição da força plantar está associada aos diferentes tipos de pés?
}

\author{
Is the distribution of plantar forces associated with different types of feet?
}

\section{Liliana Aparecida P. Azevedo¹, Luiz Fernando C. Nascimento²}

\section{RESUMO}

Objetivo: Quantificar as forças e mensurar o arco longitudinal medial em pés de escolares.

Métodos: Estudo transversal envolvendo escolares da primeira série do ensino fundamental de uma escola pública de Guaratinguetá (SP). Para identificar a distribuição das pressões plantares, foram utilizadas duas plataformas de força e a impressão plantar em papel para mensurar o arco longitudinal medial. Compararam-se as forças plantares da porção medial e lateral e as forças plantares da região anterior e posterior dos pés. Os pés, depois de classificados, foram comparados quanto às médias das forças plantares de cada sensor. A aquisição da atividade baropodométrica foi coletada três vezes seguidas para cada sensor e realizada na posição ortostática. Os valores médios foram comparados através dos testes $t$ de Student e ANOVA.

Resultados: Participaram do estudo 57 escolares com idade média de 7,5 anos sem qualquer queixa ou indício de doença ortopédica e/ou neurológica. As forças plantares foram significativamente maiores da região medial do pé esquerdo $(p=0,003)$ e em ambos os retropés $(p<0,001)$. A amostra apresentou prevalência significativamente maior de pés planos, com $59,4 \%$, pés normais com $32,1 \%$ e pés cavos com $8,5 \%$.

Conclusões: Foi possível quantificar as forças plantares, classificar os tipos de pés e associar a distribuição plantar ao tipo de pé, demonstrando que há associação entre o padrão de pé e a distribuição das forças plantares.

Palavras-chave: pé plano; saúde escolar; crianças; pressão plantar.

Instituição: Faculdade de Engenharia de Guaratinguetá da Universidade Estadual Paulista "Júlio de Mesquita Filho" (Unesp), Guaratinguetá, SP, Brasil

'Mestre em Engenharia Mecânica com ênfase em Engenharia Semiológica pela Unesp, Guaratinguetá, SP, Brasil

2Doutor em Saúde Pública pela Universidade de São Paulo; Professor do Departamento de Medicina da Universidade de Taubaté (Unitau), Taubaté, SP, Brasil

\section{ABSTRACT}

Objective: To quantify the distribution of plantar forces and to measure the foot arch of school children.

Methods: This cross-sectional study enrolled school children of the first year from a public elementary school of Guaratinguetá, SP, Brazil. In order to identify the distribution of plantar forces, two platforms of force were used and foot printing in paper was obtained to measure the foot medial arch. The plantar force was compared in medial and lateral sites and in anterior and posterior sites of the feet. The feet were compared regarding the average plantar forces in each sensor. The baropodometric acquisition was performed three times for each sensor in orthostatic position. The average values were compared by Student's $\mathrm{t}$ and ANOVA tests.

Results: 57 students with mean age of 7.5 years old were enrolled. None of them had any orthopedic or neurological problem. The plantar forces were significantly higher in the medial site of left feet $(p=0.003)$ and in the posterior site of both feet $(p<0.001)$. In the studied population, the following prevalences were found: $59.4 \%$ of flatfeet, $32.1 \%$ of normal feet and $8.5 \%$ of cavus feet.

Conclusions: It was possible to quantify the plantar forces, to classify the types of feet and to associate the plantar distribution with the type of foot. There was a relationship between the type of foot and the distribution of the plantar forces.

Key-words: flatfoot; school health; children; plantar pressure.
Endereço para correspondência:

Luiz Fernando C. Nascimento

Rua Durval Rocha, 500

CEP 12515-710 - Guaratinguetá/SP

E-mail: Ifcn@unitau.br

Fonte financiadora: Coordenação de Aperfeiçoamento de Pessoal de Nível Superior (CAPES)

Recebido em: 22/10/08

Aprovado em: 12/3/09 


\section{Introdução}

A distribuição de pressão na superfície plantar pode revelar informações tanto sobre a estrutura e função dos pés, como sobre o controle postural de todo o corpo, em condições saudáveis ou patológicas ${ }^{(1)}$. Diversos autores têm utilizado esse método de avaliação da força plantar (baropodometria) para avaliar problemas clínicos dos pés, identificar anormalidades e analisar o pós-operatório, verificando-se as mudanças na distribuição da pressão. Tal método é considerado seguro e ajuda no planejamento e tratamento dos pés ${ }^{(2)}$. No entanto, os aparelhos utilizados são caros para a realidade brasileira.

Apenas mais recentemente, a disponibilidade de transdutores de força e sistemas de aquisição de dados modernos permitiu a construção de vários sistemas de medida de distribuição da força plantar a um custo menor. Atualmente, os métodos são baseados nos efeitos das propriedades elétricas dos sensores causadas pela deformação mecânica que ocorre nos transdutores de força ${ }^{(3)}$. As plataformas de força que utilizam algumas dessas tecnologias medem, por meio do contato dos pés, a força de reação vertical do solo, que é uma força exercida abaixo da área do pé, sendo igual e oposta a todas as forças que agem de cima para baixo sobre o corpo humano ${ }^{(4)}$.

Há controvérsias sobre a acessibilidade, maneira e manuseio para o estudo dos arcos plantares dos pés de crianças e adultos, pois as técnicas de classificação costumam ter custo elevado ${ }^{(5)}$. O registro da sola dos pés ou a análise da impressão plantar no papel é um método barato, simples e viável para estimar o arco longitudinal medial dos pés de crianças e adultos ${ }^{(6)}$. As deficiências anatômicas em toda a extremidade inferior podem resultar em alinhamento e padrões de movimentos anormais do pé e tornozelo, que levam a estresse e sobrecarga excessivos sobre os tecidos moles e estruturas ósseas, dando origem a microtraumas cumulativos e dores musculoesqueléticas, com potencial para afetar a função e causar incapacidades diversas ${ }^{(7)}$.

Nesse contexto, o presente estudo tem por objetivo estimar as forças plantares, mensurar o arco longitudinal medial e avaliar a possível associação entre esses parâmetros em escolares na primeira série do ensino fundamental.

\section{Métodos}

Trata-se de um estudo transversal com alunos de uma escola da rede pública municipal da primeira série do ensino fundamental da cidade de Guaratinguetá, que se localiza a 174 km da cidade de São Paulo. Esta escola foi eleita para o estudo por ser localizada próximo ao campus universitário. Faziam parte de sua estrutura pedagógica três salas do primeiro ano do ensino fundamental. A amostra de participantes deste estudo foi de conveniência, contemplando as três séries escolares.

Foram incluídas crianças de ambos os sexos. Os pais ou responsáveis pelos alunos concordaram, por meio de assinatura do termo de consentimento livre e esclarecido e da carta de informação ao sujeito da pesquisa, com a participação de seus filhos no estudo. Foram excluídas crianças com problemas ortopédicos ou neurológicos e que tivessem sido submetidas a cirurgias ortopédicas.

As crianças foram pesadas e medidas em uma balança antropométrica da marca Welmy ${ }^{\circledR}$ (Santa Bárbara D’Oeste, Brasil), com precisão de $100 \mathrm{~g}$ para o peso e $0,5 \mathrm{~cm}$ para altura. Os pesos foram obtidos em quilogramas $(\mathrm{kg})$ e a altura em metros (m).

Para a aquisição de dados, o escolar permanecia à frente das plataformas, posicionando os pés sobre elas. Iniciada a coleta, ele levantava-se e permanecia sobre as plataformas durante 20 segundos e, em seguida, sentava-se novamente. Para todos os sensores, as medidas da força plantar foram realizadas três vezes seguidas.

Para o procedimento experimental, foram utilizadas duas plataformas de força: uma para o pé direito e outra para o esquerdo. Essas plataformas foram desenvolvidas por meio de um projeto de pesquisa específico desenvolvido no próprio Departamento de Mecânica da Faculdade de Engenharia de Guaratinguetá (FEG) da Universidade Estadual Paulista "Júlio de Mesquita Filho" (Unesp).

Cada plataforma possui 16 vigas prismáticas e a extremidade livre de cada viga corresponde a um ponto específico do pé ${ }^{(8)}$. Por meio de um programa computacional, efetuou-se com precisão e segurança a calibração automática do sinal proveniente da ponte montada com os extensômetros em $\mathrm{mV} / \mathrm{V}$ para sinal de força em Newton. Foram utilizados oito dos 16 sensores existentes em cada plataforma devido ao tamanho menor dos pés das crianças em relação a adultos.

As medidas foram coletadas dos sensores 1, 2, 3, 4 e depois $5,6,7,8$ de cada pé. Tais sensores foram selecionados após ter sido verificada a melhor combinação da posição dos sensores com a face plantar da criança (Figura 1).

Finalizada essa coleta, foi feita a documentação das impressões plantares das crianças para a classificação dos arcos plantares. Os pés das crianças foram pintados com auxílio de um rolo pequeno embebido com tinta guache de cor verde 
e solicitou-se que elas ficassem em pé sobre uma folha de papel sulfite branca.

Após a coleta dos dados, foi realizada a análise descritiva da amostra. Os valores mínimos, máximos, médios e desvios padrões da idade, peso, altura e índice de massa corpórea (IMC) foram obtidos. Utilizou-se o Índice de $\mathrm{Cole}^{(9)}$, no qual o IMC é relacionado à idade e ao sexo da criança para a classificação nutricional. Na idade de 7,5 anos, considerou-se sobrepeso se IMC estivesse acima de 18,2 kg/ $\mathrm{m}^{2}$ para meninos e $18 \mathrm{~kg} / \mathrm{m}^{2}$ para meninas; por outro lado, considerou-se obesidade IMC acima de $21,1 \mathrm{~kg} / \mathrm{m}^{2}$ para meninos e $21 \mathrm{~kg} / \mathrm{m}^{2}$ para meninas.

Estimou-se a média dos valores obtidos para cada uma das três medidas de força plantar realizadas em cada sensor. Dessas médias, selecionou-se um intervalo de tempo de cinco segundos, tempo em que as crianças estiveram estáveis sobre a plataforma de força. A partir desse intervalo, foi estimada uma média utilizada para a análise e comparação de dados.

Para avaliar em qual região do pé os sujeitos apresentaram maior ou menor força, cada plataforma foi dividida em duas partes: plano sagital em lateral ou medial e, então, plano frontal em anterior e posterior. Os sensores 1, 3, 5 e 7 corresponderam à região lateral do pé, e os sensores 2, 4, 6 e 8 representavam a região medial do pé. Com o somatório desses sensores, obtiveram-se as forças aplicadas nas laterais. Foram então estimadas e comparadas entre si as médias e os desvios padrão.

No plano frontal, a força plantar foi dividida em anterior e posterior. As médias do antepé foram estimadas com o somatório dos valores dos sensores 5, 6, 7 e 8. O retropé correspondeu ao somatório dos valores dos sensores 1, 2, 3 e 4. Foram comparadas as médias e os desvios padrão do antepé e retropé entre os pés direito e esquerdo.

As impressões plantares foram classificadas segundo o método de classificação descrito por Cavanagh e Rodgers ${ }^{(10)}$. Assim, nas impressões plantares de todos os escolares foram realizados traçados geométricos, dividindo-as em três partes. Em seguida, elas foram "escaneadas" e transferidas para o software AutoCAD $2004^{\circledR}$. A partir dos resultados das áreas, os arcos plantares foram classificados. Após confirmação dos diferentes tipos de pés encontrados na amostra, foram comparadas as forças plantares médias de todos os 16 (oito em cada um dos pés) sensores com os diferentes tipos de pés, buscando-se verificar a prevalência de maior ou menor força plantar média em alguns dos sensores em um ou outro tipo de pé.

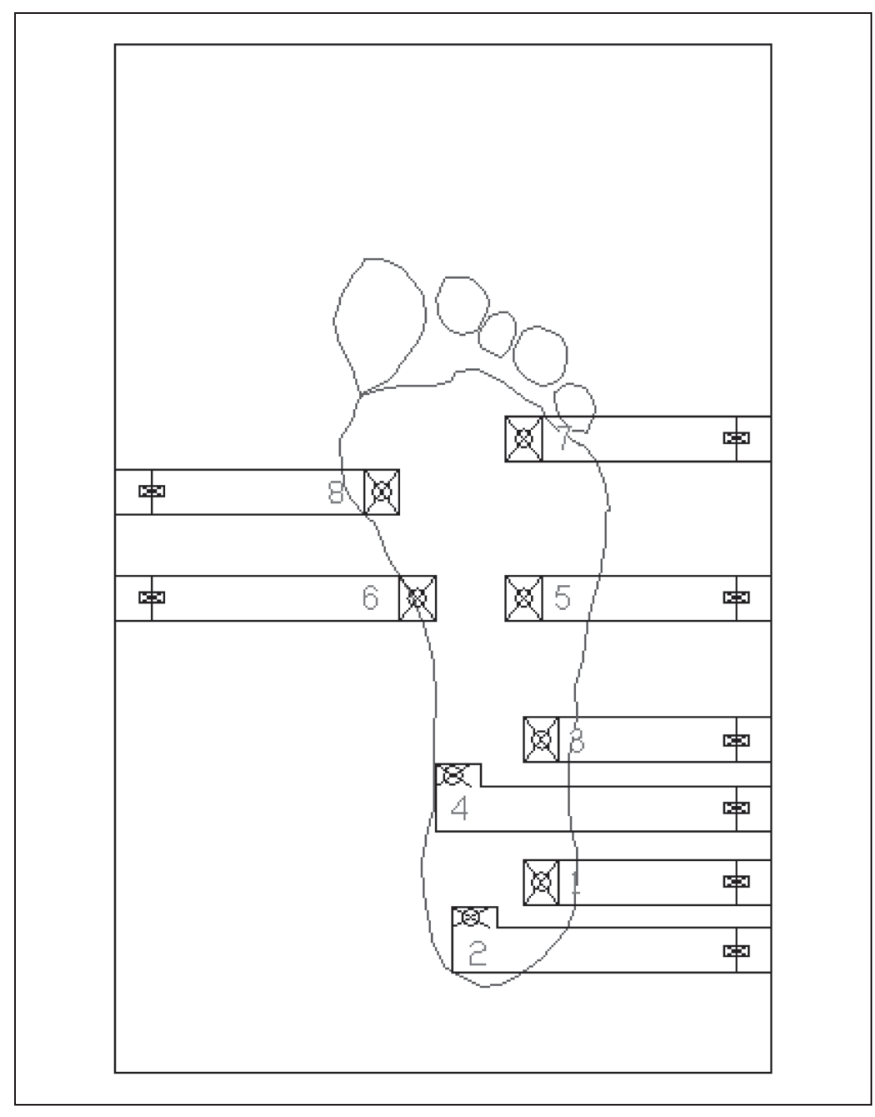

Figura 1 - Representação esquemática da disposição das vigas e pontos de aplicação da força peso para crianças de sete anos.

Essa comparação foi realizada por Análise de Variância e, quando necessário, pelo teste não paramétrico de KruskalWallis. Esse teste foi também aplicado para comparar os parâmetros antropométricos de acordo com os diferentes tipos de pés. Para comparar as médias dos resultados obtidos com os pés direito e esquerdo, utilizaram-se os testes $t$ de Student, ANOVA ou Kruskal-Wallis. Em todas as análises, considerou-se significante $p<0,05$.

\section{Resultados}

Havia 63 alunos matriculados no primeiro ano do ensino fundamental, mas incluíram-se no estudo 57 escolares, cujos pais concordaram com a participação: 30 meninas (53\%) e 27 meninos (47\%). Não houve exclusão de alunos. Observa-se, na Tabela 1, que a idade mínima foi de seis anos e 11 meses e a máxima, de nove anos e um mês, com média de 7,5 anos. Os escolares apresentaram peso médio $26,2 \mathrm{~kg}$ (variação: 18,8-41,5kg), altura média de 1,26m (variação: 1,17-1,36m) e IMC médio de $16,3 \mathrm{~kg} / \mathrm{m}^{2}$ (variação: $12,4-25,7 \mathrm{~kg} / \mathrm{m}^{2}$ ). De 
Tabela 1 - Dados demográficos dos 57 escolares estudados em Guaratinguetá

\begin{tabular}{lcccc}
\hline & Média & dp & Mínimo & Máximo \\
\hline Idade $($ meses $)$ & 91,5 & 4,7 & 83 & 109 \\
Peso $(\mathrm{kg})$ & 26,2 & 5,1 & 18,8 & 41,5 \\
Altura $(\mathrm{m})$ & 1,26 & 0,05 & 1,17 & 1,36 \\
IMC $\left(\mathrm{kg} / \mathrm{m}^{2}\right)$ & 16,3 & 2,3 & 12,4 & 25,7 \\
\hline
\end{tabular}

IMC: índice de massa corpórea; dp: desvio padrão

Tabela 2 - Força média das várias regiões dos pés dos 57 escolares de Guaratinguetá

\begin{tabular}{lrrc}
\hline & Média & \multicolumn{1}{c}{ dp } & Valor de $\boldsymbol{p}$ \\
\hline Lateral direita & 13,97 & 7,11 & $<0,001$ \\
Medial direita & 11,45 & 8,34 & \\
Lateral esquerda & 13,51 & 7,55 & NS \\
Medial esquerda & 14,52 & 13,20 & \\
Lateral direita & 13,97 & 7,11 & NS \\
Lateral esquerda & 13,51 & 7,55 & \\
Medial direita & 11,45 & 8,34 & 0,003 \\
Medial esquerda & 14,52 & 13,20 & \\
Posterior direito & 14,57 & 9,02 & $<0,001$ \\
Anterior direito & 10,89 & 6,09 & \\
Posterior esquerdo & 16,48 & 13,55 & $<0,001$ \\
Anterior esquerdo & 11,56 & 5,75 & \\
Posterior direito & 14,57 & 9,09 & NS \\
Posterior esquerdo & 16,48 & 13,55 & \\
Anterior direito & 10,89 & 6,09 & NS \\
Anterior esquerdo & 11,56 & 5,75 & \\
Média direito & 12,68 & 7,86 & 0,03 \\
Médio esquerdo & 14,02 & 10,75 & \\
\hline
\end{tabular}

NS: não significativo; dp: desvio padrão

Tabela 3 - Força detectada nos sensores 3E, 3D, 6E e 7E nos diferentes tipos de pés em Newton, nos 53 escolares de Guaratinguetá

\begin{tabular}{ccccc}
\hline & $\begin{array}{c}\text { Pés } \\
\text { planos }\end{array}$ & $\begin{array}{c}\text { Pés } \\
\text { normais }\end{array}$ & Pés cavos & $\begin{array}{c}\text { Valor } \\
\text { de } \boldsymbol{p}\end{array}$ \\
\hline 3E & $8,93(4,87)$ & $6,94(3,71)$ & $1,20(0,83)$ & 0,001 \\
3D & $9,92(6,08)$ & $3,55(2,45)$ & $1,25(1,89)$ & 0,001 \\
6E & $7,33(4,80)$ & $4,94(1,76)$ & $3,20(0,83)$ & 0,028 \\
$7 E$ & $13,10(4,18)$ & $14,50(6,44)$ & $20,20(4,43)$ & 0,02 \\
\hline
\end{tabular}

acordo com o Índice de Cole ${ }^{(9)}$, oito (14\%) escolares tinham sobrepeso e três $(5 \%)$ eram obesos.

O estudo dos arcos plantares (114 pés de 57 crianças), por meio das impressões plantares em papel, mostrou maior número de pés planos 68 (59\% dos 114 pés); 36 pés normais (32\% da amostra) e dez pés cavos (9\%).

Não houve diferença das médias das forças plantares de cada sensor entre os escolares de sexo feminino e masculino. $\mathrm{Na}$ comparação das médias das forças plantares lateral e medial (Tabela 2), observou-se que a força plantar lateral direita foi $13,97 \mathrm{~N}$ e a medial direita $11,45 \mathrm{~N}(p<0,001)$, enquanto a força plantar medial esquerda foi $14,52 \mathrm{~N}$ e a medial direita foi $11,45 \mathrm{~N}(p=0,003)$. As comparações das forças plantares da lateral esquerda com a medial esquerda e das laterais esquerda e direita não apresentaram diferenças significantes.

Percebeu-se, ainda, maior força plantar na região posterior do pé direito $(14,57 \mathrm{~N})$ do que na anterior direita $(10,89 \mathrm{~N}$; $p<0,001)$, assim como força plantar na região posterior do pé esquerdo maior $(16,48 \mathrm{~N})$ do que na anterior esquerda $(11,56 \mathrm{~N} ; p<0,001)$. As médias das forças plantares das regiões posteriores e anteriores dos lados direito e esquerdo não apresentaram diferenças significativas. Observou-se que, em geral, as crianças colocaram maior força no lado esquerdo do corpo $(14,02 \mathrm{~N})$ do que no direito $(12,68 \mathrm{~N} ; p=0,03)$.

Dos oito escolares com sobrepeso, seis apresentaram pés planos. Os três casos de obesidade, todos apresentaram pés planos. As outras variáveis, como peso e altura isoladamente, idade e sexo, não apresentaram relação com a classificação plantar, tampouco com a distribuição da força plantar.

Com relação à comparação das médias das forças plantares de todos os 16 sensores com os diferentes tipos de pés, os sensores de número 3 e 6 do lado esquerdo e 3 do lado direito apresentaram valores médios maiores nos pés planos (Tabela 3). O sensor 7 do lado esquerdo foi o único que apresentou força plantar maior nos pés cavos do que outros tipos de pés. Todos os outros doze sensores não apresentaram associação com o tipo de pé do escolar.

\section{Discussão}

Esta pesquisa estimou a distribuição da pressão plantar, mensurou o arco longitudinal medial e relacionou as variáveis em uma amostra de escolares brasileiros.

As médias das medidas antropométricas coletadas são superiores às médias encontradas por Rodriguez et al ${ }^{(11)}$, em estudo realizado com crianças brasileiras. Isso pode 
estar relacionado ao processo de aumento progressivo das medidas antropométricas de crianças e adolescentes que vem ocorrendo no Brasil nas últimas décadas. $\mathrm{O}$ percentual de obesidade e sobrepeso encontrado em nosso estudo foi 19\% (11 casos). Desses 11 casos, nove escolares apresentaram pés planos (82\%), sendo que esses 11 casos representaram $17 \%$ de todos os casos de pés planos encontrados na pesquisa, e todos os pacientes obesos apresentaram pés planos. Tais dados corroboram os achados de Bordin $e t a^{(12)}$ que estudaram a obesidade e o sobrepeso durante o desenvolvimento infantil e os relacionaram ao dismorfismo dos pés. A obesidade e o sobrepeso podem ser fatores etiológicos ou predisporem a criança a desenvolver o pé plano pelo excesso de carga sobre os pés ${ }^{(12)}$. Além desta sobrecarga, o acúmulo de gordura na borda medial dos pés, característico da infância, provavelmente tem seu volume aumentado nas crianças com sobrepeso e obesidade, favorecendo o aumento da imagem da borda medial na impressão plantar obtida.

Os valores das forças plantares obtidos neste trabalho foram utilizados para detectar a distribuição da carga corporal nos pés das crianças, trazendo importante contribuição para o levantamento do padrão dos pés. Os pacientes apresentaram maior intensidade de força na porção medial do lado esquerdo. Cavanagh et $a l^{(13)}$ supõem que essas diferenças possam refletir assimetrias posturais. Entretanto, não parece que essa suposição se aplique a esta casuística pela significativa diferença encontrada entre as porções mediais dos pés direito e esquerdo. $\mathrm{O}$ fato de ter sido encontrada maior intensidade de forças do lado medial esquerdo pode estar associado ao posicionamento das crianças durante a aquisição dos dados baropodométricos. Com interesse em perceber os seus próprios gráficos, as crianças rodaram a cabeça para o lado direito em direção ao monitor, sendo possivelmente essa a causa da maior prevalência de pressão à esquerda. Em resposta à rotação de cabeça, ocorre desestabilização postural, e a reconquista do equilíbrio envolve a necessidade de ativar músculos do lado oposto do movimento realizado. Essas respostas são ativadas em reação às informações visuais e vestibulares, justificando-se a maior intensidade de forças do lado esquerdo do corpo ${ }^{(14)}$.

Os valores estimados na divisão dos pés em retropé e antepé mostraram maior força plantar em ambos os retropés em comparação aos antepés, sem significância estatística quando comparados entre si. Betts et a ${ }^{(15)}$ apresentaram dados da relação do pico de pressão, mensurados em 29 crianças normais na posição em pé, e estimaram que a pressão no calcanhar é 1,94 vezes maior do que a pressão no antepé; no presente estudo, a relação encontrada foi de aproximadamente 1,4 vezes. Esses achados podem estar relacionados ao fato de a projeção da linha do centro de gravidade no solo, no plano sagital, passar pela articulação do tornozelo e, portanto, na porção posterior dos pés de um corpo equilibrado ou bem compensado.

A análise da impressão plantar ainda é a maneira mais utilizada por vários autores para mensurar o arco longitudinal medial e classificar os diferentes tipos de pés, embora ainda existam controvérsias sobre o uso dessa técnica ${ }^{(6)}$. Se compararmos as técnicas diretas de medidas antropométricas dos pés para mensurar a altura do arco longitudinal medial, a radiografia dos pés e o método indireto da imagem da impressão plantar, o último apresenta vantagens por prover informações sobre o arco plantar e suas anormalidade, por apresentar baixo custo e ser facilmente disponível e livre de radiação ${ }^{(16)}$.

Devido ao crescimento, na infância há uma larga variação das dimensões e formas dos pés, o que dificulta estabelecer os limites de normalidade do arco plantar nas crianças $^{(5)}$. Lin et $a^{(17)}$, em estudo realizado para tentar elucidar os fatores associados e a importância clínica do pé plano flexível, perceberam uma diminuição percentual progressiva do grau do pé plano com o aumento da idade. Os autores relacionaram este fato à evolução do desenvolvimento geral da criança (altura, peso, frouxidão ligamentar) e à realização ou não de atividade física.

A significância clínica do pé plano é uma grande preocupação para os pais e não deve ser subestimada. Além disso, o pé plano não deve ser visto somente como um problema estático do tornozelo de um pé complexo, mas também como uma anormalidade da dinâmica funcional dos membros inferiores ${ }^{(17)}$. Os dados deste estudo, no que se refere à mensuração do arco plantar e à classificação dos tipos de pés, tornaram possível observar a prevalência de pés planos (59\%), seguidos por pés normais (32\%) e pés cavos (9\%). A prevalência dos pés planos provavelmente está associada ao desenvolvimento infantil e aos casos de sobrepeso e obesidade, que representaram quase $20 \%$ da casuística. É importante destacar que os pés cavos, embora pareçam pouco frequentes, apontam um problema relevante, pois esse tipo de dismorfismo plantar costuma ser encontrado somente no início da adolescência, além de ser altamente doloroso e apresentar grande potencial para cirurgias corretivas.

Quando comparados todos os 16 sensores com os diferentes tipos de pés, dois sensores localizados na porção lateral do 
retropé dos lados esquerdo e direito e um sensor localizado na porção medial do antepé esquerdo apresentaram maior força plantar nos pés planos. Se considerarmos somente os dados do lado esquerdo, no qual as crianças puseram maior força, fica evidente um padrão de pé plano com dois pilares de força: um no antepé medial, bem na porção do desabamento do arco longitudinal medial plantar, e outro pilar de força no retropé lateral. Talvez essa distribuição das forças plantares nos pés planos promova um equilíbrio compensatório para o desabamento do arco plantar, um pilar de força em antepé medial e outro em retropé lateral.

Ainda na comparação dos 16 sensores com os diferentes tipos de pés, um sensor localizado na região lateral do antepé esquerdo mostrou força plantar maior nos pés cavos do que nos pés normais e planos. Esse achado parece condizente com o padrão de pés cavos, pois a elevação do arco plantar não permite o contato da região medial dos pés com o chão

\section{Referências bibliográficas}

1. Amadio AC, Sacco IC. Considerações metodológicas da biomecânica para a avaliação da distribuição da pressão plantar. Diabetes Clin 1999;3:42-9.

2. Stebbins JA, Harrington ME, Giacomozzi C, Thompson N, Zavatsky A, Theologis TN. Assessment of sub-division of plantar pressure measurement in children. Gait Posture 2005;22:372-6.

3. Henning EM. The evolution and biomechanics of the human foot - applied research for footwear. Rev Bras Biomec 2003;4 (Suppl 1):7-14.

4. Shumway-Cook A, Woollacott MH. Controle postural. In: Shumway-Cook A, Woolacott MFI, editors. Controle motor: teoria e aplicações práticas. $2^{\text {nd }}$ ed. São Paulo: Manole; 2003. p. 153-78.

5. Gilmour JC, Burns Y. The measurement of the medial longitudinal arch in children. Foot Ankle Int 2001;22:493-8.

6. Kanatli $U$, Yetkin H, Cila E. Footprint and radiographic analysis of the feet. J Pediatr Orthopaedics 2001;21:225-8.

7. Tokars E, Motter AA, MoroAR, Gomes ZC. A influência do arco plantar na postura e no conforto dos calçados ocupacionais. Fisioterapia Brasil 2003;4:157-62.

8. Teodoro EC, Tomazini JE, Nascimento LF. Hálux valgo e pés planos: as forças plantares são iguais? Acta Ortop Bras 2007;15:242-5.

9. Cole TJ, Bellizzi MC, Flegal KM, Dietz WH. Establishing a standard definition for child overweight and obesity worldwide: international survey. BMJ 2000;320:1240-3 e, teoricamente, empurra as forças plantares para as regiões laterais dos pés, na lateral do antepé.

Quando comparadas as médias das forças plantares de todos os sensores e a classificação dos pés entre o sexo masculino e feminino, não foi encontrada qualquer associação entre tais variáveis. Portanto, neste estudo, o sexo das crianças não foi determinante para as forças plantares nem para os diferentes tipos de pés.

Pode-se apontar como possível limitação desta investigação o tamanho da amostra envolvendo escolares do primeiro ano do ensino fundamental, que se deveu à logística do estudo, pois era necessário o transporte dos pacientes para o laboratório onde foram feitas as estimativas. Apesar disso, os resultados da pesquisa permitiram estimar a distribuição das forças plantares, mensurar o arco longitudinal medial e classificar os tipos de pés, além de associar a distribuição das forças plantares aos tipos de pés nas crianças analisadas.
10. Cavanagh PR, Rodgers MM. The arch index: a useful measure from footprints J Biomech 1987;20:547-51.

11. Rodriguez MD, Serrão JC, Ávila AO, Amadio AC. Aspectos antropométricos do pé humano: procedimentos de mensuração e relação com o crescimento na segunda infância. Rev Bras Postura Movimento 1998;2:15-27.

12. Bordin D, De Giorgi G, Mazzocco G, Rigon F. Flat and cavus foot, indexes of obesity and overweight in a population of primary-school children. Minerva Pediatr 2001;53:7-13.

13. Cavanagh PR, Rodgers MM, Liboshi A. Pressure distribution under symptomfree feet during barefoot standing. Foot Ankle 1987;7:262-76.

14. Allum JH, Pfaltz CR. Visual and vestibular contributions to pitch stabilization in the ankle muscles of normals and patients with bilateral peripheral vestibular deficits. Exp Brain Res 1985;58:82-94.

15. Betts RP, Franks Cl, Duckworth T, Burke J. Static and dynamic foot-pressure measurements in clinical orthopaedics. Med Biol Eng Comp 1980;18: 674-84.

16. Lin CH, Lee HY, Chen JJ, Lee HM, Kuo MD. Development of a quantitative assessment system for correlation analysis of footprint parameters to postural control in children. Physiol Meas 2006;27:119-30.

17. Lin CJ, Lai KA, Kuan TS, Chou YL. Correlating factors and clinical significance of flexible flatfoot in preschool children. J Pediatr Orthop 2001;21:378-82. 